\title{
A type 1 diabetes subgroup with a female bias is characterised by failure in tolerance to thyroid peroxidase at an early age and a strong association with the cytotoxic T-lymphocyte-associated antigen- 4 gene
}

\author{
J. M. M. Howson • D. B. Dunger • S. Nutland • \\ H. Stevens • L. S. Wicker • J. A. Todd
}

Received: 8 November 2006 / Accepted: 27 December 2006 / Published online: 14 February 2007

(C) Springer-Verlag 2007

\begin{abstract}
Aims/hypothesis HLA haplotypes $D R B 1^{*} 03 \_D Q B 1^{*} 02$ and $D R B 1 * 04 \_D Q B 1 * 0302$, and allelic variation of the $\mathrm{T}$ cell regulatory gene cytotoxic T-lymphocyte-associated antigen-4 (CTLA4) and of the $\mathrm{T}$ cell activation gene protein tyrosine phosphatase, non-receptor type 22 (lymphoid) (PTPN22) have been associated with type 1 diabetes and autoimmune thyroid disease. Using thyroid peroxidase autoantibodies (TPOAbs) as an indicator of thyroid autoimmunity, we assessed whether the association of these loci is different in type 1 diabetes patients with TPOAbs than in those without. Materials and methods TPOAbs were measured in 4,364 type 1 diabetic patients from across Great Britain, $67 \%$ of whom were aged under 18 years. These patients and 6,866 geographically matched control subjects were genotyped at CTLA4, PTPN22, HLA-DRB1 and HLA-DQB1.

Results TPOAbs were detected in $462(10.6 \%)$ of the type 1 diabetic patients. These patients had a stronger association with CTLA4 (odds ratio [OR] $=1.49$ for the $\mathrm{G}$ allele of the single nucleotide polymorphism rs3087243; 95\% CI $=1.29$ 1.72) than did the TPOAbs-negative patients $(p=0.0004$; $\mathrm{OR}=1.16 ; 95 \% \mathrm{CI}=1.10-1.24$ ) or type 1 diabetes patients
\end{abstract}

J. M. M. Howson - S. Nutland $\cdot$ H. Stevens $\cdot$ L. S. Wicker $\cdot$

J. A. Todd $(\bowtie)$

Juvenile Diabetes Research Foundation/Wellcome Trust Diabetes

and Inflammation Laboratory, Cambridge Institute for Medical

Research, University of Cambridge,

Wellcome Trust/MRC Building, Addenbrooke's Hospital,

Cambridge CB2 0XY, UK

e-mail: john.todd@cimr.cam.ac.uk

D. B. Dunger

Department of Paediatrics, University of Cambridge,

Addenbrooke's Hospital,

Cambridge, UK overall $(\mathrm{OR}=1.20 ; 95 \% \mathrm{CI}=1.13-1.27)$. The ratio of women:men was higher (1.94:1) in this subgroup than in type 1 diabetes patients without TPOAbs $(0.94: 1 ; p=1.86 \times$ $\left.10^{-15}\right)$. TPOAbs status did not correlate with age at diagnosis of type 1 diabetes or with PTPN22 (Arg620Trp; rs2476601). Conclusions/interpretation Our results identify a subgroup of type 1 diabetic patients that is sensitive to allelic variation of the negative regulatory molecule CTLA-4 and indicate that TPOAbs testing could be used to subclassify type 1 diabetes patients for inclusion in genetic, biological or clinical studies.

Keywords Autoimmunity $\cdot$ CTLA- 4 - Diabetes $\cdot$ Human . Thyroid antibodies
Abbreviations
AITD autoimmune thyroid disease
APS autoimmune polyendocrine syndrome
CTLA-4 cytotoxic T-lymphocyte-associated antigen-4
liCTLA4 ligand-independent form of CTLA-4
NOD non-obese diabetic
OR odds ratio
PTPN22 protein tyrosine phosphatase non-receptor type 22 (lymphoid)
SNP single nucleotide polymorphism
TPOAbs thyroid peroxidase autoantibodies

\section{Introduction}

Autoimmune diseases, a failure of immune tolerance to self antigens, are strongly inherited and often co-occur in the same patients or families [1-5], probably due to 
sharing of susceptibility alleles across multiple loci [6] and to environmental factors $[7,8]$. In particular, autoimmune thyroid disease (AITD) (Graves' disease and Hashimoto thyroiditis associated with hyper- and hypothyroidism, respectively) and autoimmune type 1 diabetes are often diagnosed in the same subjects $[1,2,4,9,10]$.

In addition to associations with HLA class II alleles and haplotypes, notably $D R B 1 * 03-D Q B 1 * 02[10-12]$, and the lymphoid-specific phosphatase $\mathrm{T}$ cell activation gene, protein tyrosine phosphatase non-receptor type 22 (lymphoid) (PTPN22) [13], AITD and type 1 diabetes have been associated with the cytotoxic T-lymphocyte-associated antigen-4 gene (CTLA4) [5], which encodes a vital negative immunoregulatory receptor in $\mathrm{T}$ cell activation and expansion. The association with AITD is strong (odds ratio $[\mathrm{OR}] \sim 1.5$ ) with single nucleotide polymorphisms (SNPs) in the 3'UTR of the gene being the strongest candidates for the causal variant(s) [14]. The SNP most associated with disease is rs 3087243 (with aliases $6230 \mathrm{G}>\mathrm{A}$ and $\mathrm{CT} 60$ ) [14]. However, the effect is much smaller in type 1 diabetes (OR 1.15-1.2) [15]. Indeed, a recent study of 769 Japanese type 1 diabetes patients, 345 of which were diagnosed with AITD [16], claimed that CTLA4 is only associated with AITD and that the association with type 1 diabetes is secondary to anti-thyroid autoimmunity such that the $\mathrm{G}$ allele of the CTLA4 rs3087243 SNP does not have any direct effect on susceptibility to type 1 diabetes. However, the reported differences were not statistically significant. In three other studies, CTLA4 was reported to be associated with co-occurrence of type 1 diabetes and AITD [5, 17, 18], which is a form of autoimmune polyendocrine syndrome (APS) [2, 19].

Autoantibodies to components of the thyroid gland, including thyroid peroxidase autoantibodies (TPOAbs), often precede diagnosis of AITD [4, 20], and CTLA4 is associated with TPOAbs production [21]. In the USA, 4.8\% of individuals aged 12 to 19 years have TPOAbs [22], whereas 10 to $30 \%$ of patients with type 1 diabetes have thyroid antibodies, up to $50 \%$ of whom progress to clinical AITD [19].

We collected plasma and DNA samples from more than 4,000 patients with type 1 diabetes, mostly from paediatric clinics from across Great Britain, with a view to investigating the association of CTLA4 in type 1 diabetes patients subclassified into patients with TPOAbs and those without.

\section{Subjects and methods}

Subjects We studied 4,364 type 1 diabetes patients from Great Britain, mostly diagnosed under the age of 16 years, with a mean $( \pm \mathrm{SD})$ of $7.5( \pm 3.9)$ years. In these patients, plasma TPOAbs levels were measured. Geographically matched control subjects $(n=6,866)$ were from the 1958 British Birth Cohort [13]. Approval from the relevant ethics committee was sought before DNAs were collected and written informed consent obtained from all participants or their parents/guardians for those too young to give consent. This investigation was carried out in accordance with the principles of the Declaration of Helsinki as revised in 2000. TPOAbs in the type 1 diabetes patients was measured by ELISA (PLATO processor; Phadia [formerly Sweden Diagnostics], Milton Keynes, UK). Recombinant TPO antigen was used, standardised against the National Institute of Biological Standards and Controls standard sera $66 / 387$. Individuals were considered positive for TPOAbs above $85 \mathrm{IU} / \mathrm{ml}$.

Genotyping The rs3087243 (+6230G $>$ A) SNP in CTLA4 was genotyped in all samples because it was the variant most associated with both type 1 diabetes and AITD in a recent study [14]. Genotyping was done using TaqMan MGB chemistries (Applied Biosystems, Warrington, UK). Details of the genotyping of the SNPs of PTPN22 (Arg620Trp; rs2476601) and the insulin gene (INS) (rs689) have been described previously [13, 23]. HLA$D R B 1$ and $-D Q B 1$ were typed in a subset of these British patients using the Dynal-Roche system (Dynal Biotech, Wirral, UK).

We successfully genotyped and measured TPOAbs in type 1 diabetic patients as follows: 3,998 patients at CTLA4, 4,219 patients at INS, 4,203 patients at PTPN22, 2,034 patients at $H L A-D Q B 1$ and 1,952 patients at $H L A$ $D R B 1$. We also had genotypes for 6,866 control subjects at CTLA4.

Statistical methods Logistic regression was used to test for association and to calculate ORs, with 95\% CIs, using STATA8 (www.stata.com) and the rs3087243 A/A genotype as reference at $\mathrm{OR}=1.0$. As patients and control subjects were collected from across Great Britain, analyses were stratified according to 12 geographical regions by putting a regional variable into the regression [24]. In this way any confounding due to variation in allele frequency across Britain was minimised. To test for an interaction effect between genotype (at rs3087243, PTPN22 or INS) or haplotype (HLA-DRB1_HLA-DQB1) and the presence of TPOAbs in type 1 diabetic patients, a patient-only logistic regression approach was adopted with TPOAbs status as the outcome variable. We also tested whether the subject's sex explained the variation in TPOAbs by regressing sex on TPOAbs status. Sex was found to be an important determinant of TPOAbs status and so was included as a confounder in all analyses of TPOAbs by including a sex variable in the regression. 
Table 1 Phenotypes of the type 1 diabetic subgroups tested for thyroid autoantibodies

\begin{tabular}{|c|c|c|c|c|}
\hline & All type 1 diabetes patients & TPOAbs-positive patients & TPOAbs-negative patients & $p$ value \\
\hline Women:men $(n ; \%)$ & $0.94: 1(2,112: 2,251 ; 48.4: 51.6)$ & $1.94: 1(305: 157 ; 66.0: 34.0)$ & $0.86: 1(1,767: 2,060 ; 46.2: 53.8)$ & $1.86 \times 10^{-15}$ \\
\hline \multicolumn{5}{|c|}{ Age at diagnosis of type 1 diabetes, mean (SD) (years) } \\
\hline Overall & $7.5(3.9)$ & $7.6(4.1)$ & $7.5(3.9)$ & \multirow[t]{3}{*}{0.16} \\
\hline Male & $7.5(4.0)$ & $7.5(4.5)$ & $7.5(4.0)$ & \\
\hline Female & $7.5(3.8)$ & $7.7(3.8)$ & $7.4(3.8)$ & \\
\hline \multicolumn{5}{|c|}{ Median age at diagnosis of type 1 diabetes (years) } \\
\hline Overall & 7 & 8 & 7 & \multirow[t]{3}{*}{ NA } \\
\hline Male & 7 & 7 & 7 & \\
\hline Female & 7 & 8 & 7 & \\
\hline
\end{tabular}

NA, not applicable

\section{Results}

Thyroid autoantibodies in type 1 diabetes Of 4,364 patient samples tested, 462 (10.6\%) were TPOAbs-positive, which is similar to previous studies $[4,5]$. The ratio of women: men was significantly elevated in the TPOAbs-positive patients (1.94:1) compared with the TPOAbs-negative patients $(0.86: 1)$ or the type 1 diabetes patients overall (0.94:1) (Table 1). Female type 1 diabetes patients are more likely to have TPOAbs $(\mathrm{OR}=2.31 ; 95 \% \mathrm{CI}=1.87-2.85$; $p=1.86 \times 10^{-15}$ ) (Table 1 ). The mean age at diagnosis of diabetes did not differ between the TPOAbs-positive (7.6 years) and negative patients ( 7.5 years; $p=0.16$ ) (Table 1, Fig. 1), with median ages at type 1 diabetes diagnosis of 8 and 7 years, respectively (Table 1, Fig. 1). In the TPOAbs-positive subgroup there was no difference between females and males in the age at diagnosis of type 1 diabetes (means 7.71 and 7.50 years; ranges $1-16$ and $1-21)$. There was no effect of duration of type 1 diabetes on the presence of TPOAbs $(p=0.73)$ once age at blood donation was accounted for in the regression model.

Genetic control of type 1 diabetes with thyroid autoantibodies With regard to CTLA4, we observed a convincing

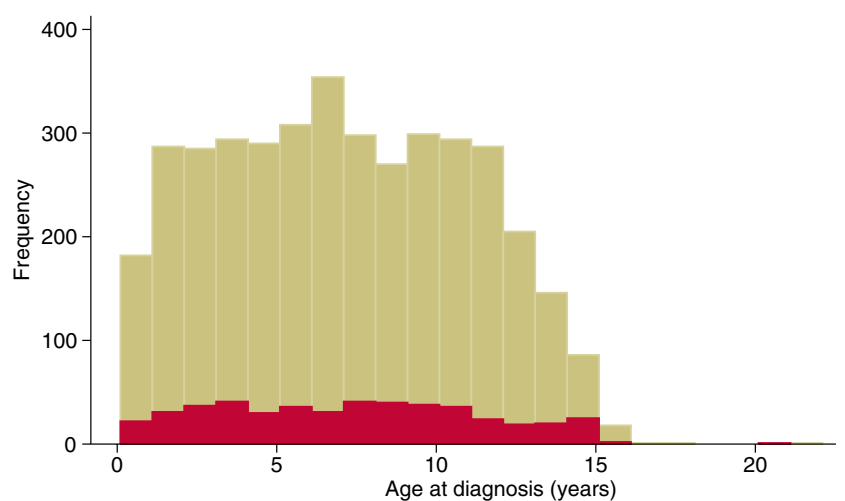

Fig. 1 Frequency distribution of age at diagnosis of type 1 diabetes by thyroid antibodies status in 1-year intervals. Beige, TPOAbsnegative; red, TPOAbs-positive difference between TPOAbs subgroups $(p=0.0004)$ (Tables 2, 3 and 4), with a $6.2 \%$ increase in the frequency of the disease-associated $G$ allele of rs3087243 in the TPOAbs-positive group (Table 2) and almost twice the risk of having both type 1 diabetes and TPOAbs associated with the $\mathrm{G}$ allele or $\mathrm{G} / \mathrm{G}$ genotype (OR of the $\mathrm{G} / \mathrm{G}$ genotype= 2.63 in the TPOAbs-positive group compared with 1.37 in the TPOAbs-negative group, with no overlap in the $95 \%$ CIs) (Table 4).

One other interesting difference observed by us in the association of CTLA4 with the TPOAbs-positive type 1 diabetes patients, was the mode of inheritance of the locus: the disease risk of the alleles did not fit with the multiplicative model, as seen in both type 1 diabetes and AITD (Table 4) ( $p=0.0097$ against this model); instead, the $\mathrm{G}$ allele had a dominant effect with no significant difference between the $\mathrm{G} / \mathrm{A}$ heterozygote $(\mathrm{OR}=2.16)$ and the $\mathrm{G} / \mathrm{G}$ homozygous genotype risks $(\mathrm{OR}=2.63)$ (Table 4$)$. Note that the CTLA4 rs3087243 $\mathrm{G}$ allele did not affect age at diagnosis of type 1 diabetes overall $(p=0.18)$ or of type 1 diabetes in TPOAbs-positive patients $(p=0.67)$, nor was it differentially associated in females and males $(p=0.61)$.

We also tested whether the other susceptibility genes established to be associated with type 1 diabetes and AITD, namely $H L A-D R B 1, H L A-D Q B 1$, INS and PTPN22, were differentially associated with the two disease subgroups (Table 2). No convincing evidence of an interaction between TPOAbs-positivity and alleles, genotypes or haplotypes of these genes was obtained (Table 2).

\section{Discussion}

Our results indicate that certain type 1 diabetic patients suffer from an immune tolerance defect to both the insulinproducing beta cell and the thyroid gland, which can occur very early in life, since TPOAbs are present in the youngest of type 1 diabetic patients (Fig. 1). This form of disease is 
Table 2 Genetic associations in thyroid autoantibodies-positive and -negative British type 1 diabetes patients

\begin{tabular}{|c|c|c|c|c|c|c|}
\hline & $\begin{array}{l}\text { Number of } \\
\text { patients }^{\mathrm{a}}\end{array}$ & $\begin{array}{l}\text { Frequency }(\%) \text { in } \\
\text { TPOAbs-positive } \\
\text { patients }\end{array}$ & $\begin{array}{l}\text { Frequency }(\%) \text { in } \\
\text { TPOAbs-negative } \\
\text { patients }\end{array}$ & $\begin{array}{l}\text { Frequency }(\%) \\
\text { in women }\end{array}$ & $\begin{array}{l}\text { Frequency } \\
(\%) \text { in } \text { men }^{b}\end{array}$ & $p_{\text {difference }}$ \\
\hline CTLA4 rs3087243 (G>A) & 3,998 & 64.5 & 58.6 & 48.6 & 51.4 & 0.0004 \\
\hline DRB1*03_DQB1*02 & 1,956 & 38.8 & 33.7 & 47.7 & 52.3 & 0.02 \\
\hline DRB1*03_DQB1*02/DRB1*03_DQB $1 * 02$ & 198 & 7.8 & 3.9 & 44.4 & 55.6 & 0.05 \\
\hline$D R B 1 * 0401 \_D Q B 1 * 0302$ & 1,956 & 20.3 & 24.9 & 47.0 & 53.0 & 0.72 \\
\hline $\mathrm{DR} 3 / 4(\operatorname{not} \bar{D} R B 1 * 0403)$ & 2,046 & 32.0 & 34.1 & 47.5 & 52.5 & 0.52 \\
\hline$D R B 1 * 15 \_D Q B 1 * 0602$ & 1,956 & 0.7 & 0.2 & 36.4 & 63.6 & 0.02 \\
\hline INS-23HphI rs689 & 4,219 & 14.9 & 15.4 & 48.4 & 51.6 & 0.63 \\
\hline PTPN22 rs2476601 & 4,203 & 19.6 & 16.9 & 48.3 & 51.7 & 0.06 \\
\hline
\end{tabular}

${ }^{\mathrm{a}}$ These are the numbers of patients with TPOAbs measures who were also genotyped at the locus listed. Sex and age at donation of blood are included in tests of statistical differences between the TPOAbs-positive and -negative subgroups.

${ }^{\mathrm{b}}$ For the $H L A-D R B 1$ and $-D Q B 1$ haplotypes, percentages are based on the most likely haplotype assignment.

strongly regulated by allelic variation in CTLA4. This conclusion is consistent with the critical role of CTLA4 in peripheral tolerance as an inhibitory regulator of $\mathrm{T}$ cell activation, $\mathrm{T}$ cell proliferation and apoptosis, as well as its roles in the functions of antigen presenting cells and $\mathrm{T}$ regulatory cells [25-27]. We have proposed that a reduced function of CTLA4 associated with the rs3087243 $\mathrm{G}$ allele allows $\mathrm{T}$ cells to be more hyperactive and to respond to peripheral antigens to a greater degree than individuals carrying the rs3087243 SNP A/A genotype, which is associated with autoimmune disease protection and increased peripheral tolerance $[14,28]$. However, our results also indicate that allelic variation of CTLA4 has a much smaller effect in approximately $90 \%$ of type 1 diabetic patients. Previous studies have reported that in excess of $15 \%$ of European type 1 diabetic patients develop antithyroid autoimmunity [19]. Thus if we had been able to detect these within our patient samples, the OR in the TPOAbs-negative subgroup would probably have decreased to 1 or very close to it. Our interpretation of this result is different from a recent Japanese study, which concluded that CTLA4 is only associated with AITD in type 1 diabetic patients [16] and that the association with type 1 diabetes is secondary to anti-thyroid autoimmunity such that the $\mathrm{G}$ allele of the CTLA4 rs3087243 SNP does not have any direct effect on susceptibility to type 1 diabetes.

We suggest that allelic variation of CTLA4 does directly affect type 1 diabetes by predisposing individuals with a $G$ allele to reduced levels of tolerance to a multiplicity of peripheral antigens and maybe even a form of APS (the occurrence in patients of two or more endocrine autoimmune diseases). We also note that in patients with both type 1 diabetes and AITD, type 1 diabetes nearly always occurs more than a decade before the development of clinical AITD. However, the strongest evidence for a direct effect of allelic variation of CTLA4 on type 1 diabetes susceptibility is provided by studies in the non-obese diabetic (NOD) mouse [14, 29-31]. Genetic analysis of the NOD mouse model of type 1 diabetes has shown that the region of mouse chromosome 1 containing Ctla 4 and the insulin dependent diabetes susceptibility 5a (Idd5a, previously known as Idd5.1) locus has a significant effect on disease susceptibility [14, 29-31]. The causal variant has been mapped to a SNP in exon 2 of Ctla4, for which the NOD allele reduces the splicing and production of an alternative mRNA isoform of CTLA4, the ligand-independent form (liCTLA4) [14]. Moreover, it has been shown that liCTLA4 has inhibitory functions in T cell activation [29].

NOD mouse studies have also shown that different combinations of alleles of multiple susceptibility genes can lead to a variety of autoimmune phenotypes, including liver disease and AITD, as well as type 1 diabetes with different rates of progression [32] (K. Hunter, J. A. Todd, L. S. Wicker, unpublished data). In particular, the effect of resistance alleles in mouse Ctla4 is masked when resistance alleles at a separate disease locus are present in the mouse

Table 3 Allele and genotype frequencies at CTLA4 rs3087243 in type 1 diabetic and Graves' patients

\begin{tabular}{|c|c|c|c|c|c|}
\hline Allele/genotype & $\begin{array}{l}\text { All type } 1 \text { diabetes patients } \\
{[n(\%)]}\end{array}$ & $\begin{array}{l}\text { TPOAbs-positive } \\
{[n(\%)]}\end{array}$ & $\begin{array}{l}\text { TPOAbs-negative } \\
{[n(\%)]}\end{array}$ & $\begin{array}{l}\text { Graves' patients } \\
{[n(\%)]}\end{array}$ & $\begin{array}{l}\text { Control subjects } \\
{[n(\%)]}\end{array}$ \\
\hline $\mathrm{G}$ & $4,819(59.3)$ & $560(64.5)$ & $4,174(58.6)$ & $835(63.4)$ & $7,523(54.8)$ \\
\hline $\mathrm{A} / \mathrm{A}$ & $663(16.3)$ & $44(10.1)$ & $611(17.1)$ & $90(13.7)$ & $1,431(20.8)$ \\
\hline $\mathrm{A} / \mathrm{G}$ & 1,987 (48.9) & $220(50.7)$ & 1,732 (48.6) & $301(45.7)$ & $3,347(48.8)$ \\
\hline $\mathrm{G} / \mathrm{G}$ & $1,416(34.8)$ & $170(39.2)$ & $1,221(34.3)$ & $267(40.6)$ & $2,088(30.4)$ \\
\hline
\end{tabular}


Table 4 Odds ratios with 95\% CIs at CTLA4 rs3087243 for all type 1 diabetic patients and by TPOAbs positivity compared with Graves' patients

\begin{tabular}{|c|c|c|c|c|c|c|c|c|}
\hline \multirow[t]{2}{*}{ Allele/genotype } & \multicolumn{2}{|c|}{ All type 1 diabetes patients } & \multicolumn{2}{|c|}{ TPOAbs-positive patients ${ }^{\mathrm{a}}$} & \multicolumn{2}{|c|}{ TPOAbs-negative patients } & \multicolumn{2}{|c|}{ Graves' disease patients ${ }^{\mathrm{b}}$} \\
\hline & OR $(95 \% \mathrm{CI})$ & $p$ value & OR $(95 \% \mathrm{CI})$ & $p$ value & OR $(95 \% \mathrm{CI})$ & $p$ value & OR $(95 \% \mathrm{CI})$ & $p$ value \\
\hline $\mathrm{G}^{\mathrm{c}}$ & $1.20(1.13-1.27)$ & $3.71 \times 10^{-10}$ & $1.49(1.29-1.72)$ & $4.08 \times 10^{-8}$ & $1.16(1.10-1.24)$ & $5.54 \times 10^{-7}$ & $1.37(1.19-1.57)$ & $6.25 \times 10^{-6}$ \\
\hline $\mathrm{A} / \mathrm{A}$ & $1.00(\mathrm{ref})$ & & $1.00(\mathrm{ref})$ & & 1.00 (ref) & & $1.00(\mathrm{ref})$ & \\
\hline $\mathrm{A} / \mathrm{G}$ & $1.30(1.16-1.45)$ & $8.66 \times 10^{-10}$ & $2.16(1.55-3.01)$ & $1.03 \times 10^{-8}$ & $1.23(1.09-1.38)$ & $2.10 \times 10^{-6}$ & $1.32(0.99-1.74)$ & 0.00004 \\
\hline $\mathrm{G} / \mathrm{G}$ & $1.47(1.31-1.66)$ & & $2.63(1.87-3.70)$ & & $1.37(1.22-1.55)$ & & $1.85(1.39-2.47)$ & \\
\hline
\end{tabular}

${ }^{\text {a }} \mathrm{A}$ two degree of freedom genotype test should be used here as the multiplicative model is inappropriate $p=0.0097$.

b In the Graves' disease analysis, the patients, who are from a previous study [14] and were not collected from all 12 sub-regions of Great Britain, were matched to 1,745 control subjects from the regions of their origin.

${ }^{\mathrm{c}}$ Allele $\mathrm{A}$ is used as reference (ref).

and when, in a separate susceptibility gene combination, the resistance allele in mouse Ctla4 can completely mask the potent disease-causing effect of a particular susceptibility allele at another locus. It has also been shown that a third locus, insulin dependent diabetes susceptibility 3 (Idd3), which is believed to be the interleukin-2 gene $[32,33]$ can influence the production of CTLA-4 [30, 34]. We, therefore, propose that AITD, type 1 diabetes and cooccurring type 1 diabetes and AITD manifest due to different, but overlapping combinations of susceptibility and resistance alleles of multiple loci, and that in isolated type 1 diabetes (up to $90 \%$ of cases) the constellations of alleles giving rise to this disease mask the allelically variable effect of CTLA4 to a much greater degree than the combination of alleles causing AITD or type 1 diabetes + TPOAbs as studied here.

It will be interesting to try to identify genes with alleles that can mask the effect of CTLA4 alleles in humans. At the same time, we cannot ignore the role of the yet-to-bedefined environmental and developmental factors in determining the penetrance of susceptibility genes. Finally, the bias in the proportion of female type 1 diabetic patients with TPOAbs is comparable with previous, small studies of 35 [5], 28 [18] and 77 [17] APS (type 1 diabetes + AITD) patients, in which the ratios of women:men were $1.7: 1,2.5: 1$ and $2.3: 1$, respectively, suggesting that $X$ linked factors are operating. One possibility is that the skewing of $\mathrm{X}$ inactivation, which has been described in AITD patients [35, 36], alters expression of immune genes on chromosome $\mathrm{X}$, thereby predisposing to autoimmunity in females. We note in our data that the bias may begin in patients with age at diagnosis of less than 10 years, in whom the ratio of women:men in TPOAbs-positive type 1 diabetes cases was 1.28:1, compared with $0.88: 1$ in the TPOAbs-negative patients, occurring before the onset of puberty and therefore at an age when hormonal effects are unlikely to influence the penetrance of APS in females.

In conclusion, our study is unique in that it shows a statistically significant difference in: (1) the association between CTLA4 and type 1 diabetes patient subgroups; and
(2) the ratio of women:men within these subgroups. Moreover, with regard to TPOAbs status, which is a routine clinical test, we have shown that the TPOAbs marker is present in many type 1 diabetic patients at a young age and could therefore be a useful and early biomarker for this subclass of type 1 diabetes. In ongoing clinical trials to reverse or retard type 1 diabetes, inter-individual genetic variation in the balance of immune effector responses and tolerance could be partially reflected by TPOAbs status, thereby making it a parameter that would positively correlate with a poor outcome for tolerance-inducing therapeutic intervention.

Acknowledgements We thank the DNA team for the preparation of DNA and plasma samples. We acknowledge the assistance of D. Strachan, M. Pembrey, W. McArdle, S. Ring and Paul Burton in this project. L. S. Wicker and J. A. Todd are supported by grants from the Juvenile Diabetes Research Foundation and the Wellcome Trust. L. S. Wicker is a Juvenile Diabetes Research Foundation/Wellcome Trust Principal Research Fellow. We acknowledge use of DNA from the 1958 British Birth Cohort collection, funded by the Medical Research Council grant G0000934 and Wellcome Trust grant 068545/Z/02.

Duality of interest The authors declare that they have no duality of interest.

\section{References}

1. Tait KF, Marshall T, Berman J et al (2004) Clustering of autoimmune disease in parents of siblings from the type 1 diabetes Warren repository. Diabet Med 21:358-362

2. Eisenbarth GS, Gottlieb PA (2004) Autoimmune polyendocrine syndromes. N Engl J Med 350:2068-2079

3. Alarcon-Segovia D, Alarcon-Riquelme ME, Cardiel MH et al (2005) Familial aggregation of systemic lupus erythematosus, rheumatoid arthritis, and other autoimmune diseases in 1,177 lupus patients from the GLADEL cohort. Arthritis Rheum 52:1138-1147

4. Barker JM, Yu J, Yu L et al (2005) Autoantibody "subspecificity" in type 1 diabetes: risk for organ-specific autoimmunity clusters in distinct groups. Diabetes Care 28:850-855

5. Golden B, Levin L, Ban Y, Concepcion E, Greenberg DA, Tomer Y (2005) Genetic analysis of families with autoimmune diabetes and thyroiditis: evidence for common and unique genes. J Clin Endocrinol Metab 90:4904-4911 
6. Wang WY, Barratt BJ, Clayton DG, Todd JA (2005) Genomewide association studies: theoretical and practical concerns. Nat Rev Genet 6:109-118

7. Todd JA (1991) A protective role of the environment in the development of type 1 diabetes? Diabet Med 8:906-910

8. Knip M, Veijola R, Virtanen SM, Hyoty H, Vaarala O, Akerblom HK (2005) Environmental triggers and determinants of type 1 diabetes. Diabetes 54(Suppl 2):S125-S136

9. Riley WJ, Maclaren NK, Lezotte DC, Spillar RP, Rosenbloom AL (1981) Thyroid autoimmunity in insulin-dependent diabetes mellitus: the case for routine screening. J Pediatr 99:350-354

10. Holl RW, Bohm B, Loos U, Grabert M, Heinze E, Homoki J (1999) Thyroid autoimmunity in children and adolescents with type 1 diabetes mellitus. Effect of age, gender and HLA type. Horm Res 52:113-118

11. Simmonds MJ, Howson JM, Heward JM et al (2005) Regression mapping of association between the human leukocyte antigen region and Graves disease. Am J Hum Genet 76:157-163

12. Einarsdottir E, Soderstrom I, Lofgren-Burstrom A et al (2003) The CTLA4 region as a general autoimmunity factor: an extended pedigree provides evidence for synergy with the HLA locus in the etiology of type 1 diabetes mellitus, Hashimoto's thyroiditis and Graves' disease. Eur J Hum Genet 11:81-84

13. Smyth D, Cooper JD, Collins JE et al (2004) Replication of an association between the lymphoid tyrosine phosphatase locus (LYP/PTPN22) with type 1 diabetes, and evidence for its role as a general autoimmunity locus. Diabetes 53:3020-3023

14. Ueda H, Howson JM, Esposito L et al (2003) Association of the T-cell regulatory gene CTLA4 with susceptibility to autoimmune disease. Nature 423:506-511

15. Howson JM, Barratt BJ, Todd JA, Cordell HJ (2005) Comparison of population- and family-based methods for genetic association analysis in the presence of interacting loci. Genet Epidemiol 29:51-67

16. Ikegami H, Awata T, Kawasaki E et al (2006) The association of CTLA4 polymorphism with type 1 diabetes is concentrated in patients complicated with autoimmune thyroid disease: a multicenter collaborative study in Japan. J Clin Endocrinol Metab 91:10871092

17. Djilali-Saiah I, Larger E, Harfouch-Hammoud E et al (1998) No major role for the CTLA-4 gene in the association of autoimmune thyroid disease with IDDM. Diabetes 47:125-127

18. Takara M, Komiya I, Kinjo Y et al (2000) Association of CTLA-4 gene $\mathrm{A} / \mathrm{G}$ polymorphism in Japanese type 1 diabetic patients with younger age of onset and autoimmune thyroid disease. Diabetes Care 23:975-978

19. Barker JM (2006) Clinical review: type 1 diabetes-associated autoimmunity: natural history, genetic associations, and screening. J Clin Endocrinol Metab 91:1210-1217

20. Kordonouri O, Klinghammer A, Lang EB, Gruters-Kieslich A, Grabert M, Holl RW (2002) Thyroid autoimmunity in children and adolescents with type 1 diabetes: a multicenter survey. Diabetes Care 25:1346-1350
21. Tomer Y, Greenberg DA, Barbesino G, Concepcion E, Davies TF (2001) CTLA-4 and not CD28 is a susceptibility gene for thyroid autoantibody production. J Clin Endocrinol Metab 86:1687-1693

22. Hollowell JG, Staehling NW, Flanders WD et al (2002) Serum TSH, T(4), and thyroid antibodies in the United States population (1988 to 1994): National Health and Nutrition Examination Survey (NHANES III). J Clin Endocrinol Metab 87:489-499

23. Barratt BJ, Payne F, Lowe CE et al (2004) Remapping the insulin gene/IDDM2 locus in type 1 diabetes. Diabetes 53:18841889

24. Clayton DG, Walker NM, Smyth DJ et al (2005) Population structure, differential bias and genomic control in a large-scale, case-control association study. Nat Genet 37:1243-1246

25. Engelhardt JJ, Sullivan TJ, Allison JP (2006) CTLA-4 overexpression inhibits $\mathrm{T}$ cell responses through a CD28-B7-dependent mechanism. J Immunol 177:1052-1061

26. Scheipers P, Reiser H (1998) Role of the CTLA-4 receptor in T cell activation and immunity. Physiologic function of the CTLA-4 receptor. Immunol Res 18:103-115

27. Eggena MP, Walker LS, Nagabhushanam V, Barron L, Chodos A, Abbas AK (2004) Cooperative roles of CTLA-4 and regulatory T cells in tolerance to an islet cell antigen. J Exp Med 199:1725-1730

28. Atabani SF, Thio CL, Divanovic S et al (2005) Association of CTLA4 polymorphism with regulatory $\mathrm{T}$ cell frequency. Eur $\mathrm{J}$ Immunol 35:2157-2162

29. Vijayakrishnan L, Slavik JM, Illes Z et al (2004) An autoimmune disease-associated CTLA-4 splice variant lacking the B7 binding domain signals negatively in $T$ cells. Immunity $20: 563-575$

30. Lundholm M, Motta V, Lofgren-Burstrom A et al (2006) Defective induction of CTLA-4 in the NOD mouse is controlled by the NOD allele of Idd3/IL-2 and a novel locus (Ctex) telomeric on chromosome 1. Diabetes 55:538-544

31. Lamhamedi-Cherradi SE, Boulard O, Gonzalez C et al (2001) Further mapping of the Idd5.1 locus for autoimmune diabetes in NOD mice. Diabetes 50:2874-2878

32. Wicker LS, Moule CL, Fraser H et al (2005) Natural genetic variants influencing type 1 diabetes in humans and in the NOD mouse. Novartis Found Symp 267:57-65 (discussion 65-75)

33. Yamanouchi J, Rainbow D, Serra P et al (2007) Interleukin-2 gene variation impairs regulatory $\mathrm{T}$ cell function and causes autoimmunity. Nat Genet DOI 10.1038/ng1958

34. Hwang KW, Sweatt WB, Mashayekhi M et al (2004) Transgenic expression of CTLA-4 controls lymphoproliferation in IL-2deficient mice. J Immunol 173:5415-5424

35. Ozcelik T, Uz E, Akyerli CB et al (2006) Evidence from autoimmune thyroiditis of skewed X-chromosome inactivation in female predisposition to autoimmunity. Eur J Hum Genet 14:791-797

36. Chitnis S, Monteiro J, Glass D et al (2000) The role of Xchromosome inactivation in female predisposition to autoimmunity. Arthritis Res 2:399-406 